\title{
THE COUPLED CLUSTER METHOD APPLIED TO THE SPIN-HALF $X X Z$ MODEL ON THE HONEYCOMB LATTICE
}

\author{
R. F. BISHOP* and J. ROSENFELD ${ }^{\dagger}$ \\ Department of Physics, UMIST, \\ P. O. Box 88, Manchester, M60 1QD, UK
}

Received 29 June 1998

\begin{abstract}
The coupled cluster method (CCM) is applied to the spin-half $X X Z$ model on the honeycomb lattice. Hierarchical CCM approximation schemes are applied in order to obtain the ground-state properties. The possible critical behavior of this model is also examined. Reasonably good results for the ground-state energy and the staggered magnetization are obtained, in comparison to the results from Monte Carlo simulations, spin-wave techniques, series expansions, and finite lattice diagonalizations, that have already been performed on this model.
\end{abstract}

\section{Introduction}

There are no conclusive results for the spin-half $X X Z$ (or anisotropic Heisenberg) model on two-dimensional lattices. A rigorous treatment by Bethe ${ }^{1}$ has only been possible for the one-dimensional case. However, there has been extensive work carried out in the particular case of the two-dimensional square lattice, using a number of techniques including the coupled cluster method (CCM) considered here. This has produced convincing, if not completely definitive, results that suggest that the square lattice has a Néel-ordered ground state. ${ }^{2}$ The situation for the honeycomb lattice is in more doubt, however, because less attention has been paid to it, and hence there are less results for comparison.

The honeycomb lattice case is a bipartite system, as the lattice can be split into two identical sub-lattices. Frustrated models, such as the triangular lattice case, do not have this property. Nevertheless, they have also been examined by the CCM. ${ }^{3}$ The honeycomb lattice also has the least possible co-ordination number of any regular two-dimensional lattice. The $1 \mathrm{D}$ chain, which has the smallest co-ordination number possible for any lattice, suffers from the greatest quantum effects, to such an extent that it has no long-range order (LRO). The co-ordination number of the honeycomb lattice is larger than that of the $1 \mathrm{D}$ chain, but smaller than that of the square lattice. Thus, the quantum fluctuations present in the honeycomb case

\footnotetext{
*E-mail: r.f.bishop@umist.ac.uk
}

$\dagger_{\text {E-mail: mcbpgjr@afs.mcc.ac.uk }}$ 
are expected to be less than those in the $1 \mathrm{D}$ chain case, but greater than those in the $2 \mathrm{D}$ square lattice case. Hence, there is an a priori possibility that quantum fluctuations may destroy antiferromagnetic (AFM) LRO in the honeycomb case.

However, from previous work done on the honeycomb lattice case, it is believed to possess a Néel-ordered ground state. Strong indications of the survival of LRO initially came from the spin-wave approximations of Anderson and Kubo ${ }^{4}$ in 1952 and $\mathrm{Oguchi}^{5}$ in 1960 , which apply to any two-dimensional lattice. More recently, a Monte Carlo simulation by Reger, Riera, and Young, ${ }^{6}$ extrapolations of finite lattice diagonalizations, performed by Oitmaa and Betts, ${ }^{7}$ series expansions around the Ising limit, ${ }^{8}$ and a spin-wave calculation by Zheng, Oitmaa, and Hamer, ${ }^{9}$ have all indicated that although quantum fluctuations on the honeycomb lattice case are certainly stronger than on the square lattice case, the system does still possess Néel order.

The CCM has already been successfully applied to the spin-half $X X Z$ model on the one-dimensional chain and square lattice ${ }^{10}$ and on the frustrated triangular lattice. $^{3}$ In this paper the CCM is applied to the honeycomb case, in order to calculate the ground-state energy and the staggered magnetization. We shall see that it also provides an indication of a phase transition in this model.

Since a detailed description of the CCM formulation has been given elsewhere, ${ }^{11}$ only the essential components for calculating the ground-state properties of a system will be given here. The CCM parametrization of the ground ket state is given by:

$$
|\psi\rangle=e^{S}|\phi\rangle ; \quad S=\sum_{I}^{\prime} s_{I} C_{I}^{\dagger} .
$$

The correlation operator, $S$, is decomposed solely in terms of the multiconfigurational creation operators, $C_{I}^{\dagger}$, over a complete set of the many-body configurations, $\{I\}$, defined by a set-index $I$ which describes the set of spins which are flipped with respect to those contained in a suitably chosen, normalised, model state $|\phi\rangle$. The prime on the sum in Eq. (1) excludes the null set, $I \rightarrow 0$, corresponding to the identity operator $C_{0}^{\dagger} \equiv \mathbb{1}$. The Schrödinger equation can be expressed as:

$$
e^{-S} H e^{S}|\phi\rangle=E_{g}|\phi\rangle \text {. }
$$

Consequently, the ground-state energy may be obtained by taking the inner product of Eq. (2) with the model state $|\phi\rangle$ :

$$
\left\langle\phi\left|e^{-S} H e^{S}\right| \phi\right\rangle=E_{g} .
$$

The coupled set of microscopic nonlinear equations for the unknown $c$-number coefficients $s_{I}$ may be obtained by taking the inner product of Eq. (2) with the complete set of states $\left\{C_{I}^{\dagger}|\phi\rangle\right\}$ :

$$
\left\langle\phi\left|C_{I} e^{-S} H e^{S}\right| \phi\right\rangle=0 ; \quad I \neq 0 .
$$

The similarity transformed Hamiltonian, $e^{-S} H e^{S}$, may be expressed as the following nested commutator expansion:

$$
e^{-S} H e^{S}=H+[H, S]+\frac{1}{2 !}[[H, S], S]+\cdots
$$


This expansion always terminates at finite order, as a consequence of the correlation operator being composed solely of creation operators, provided only that the Hamiltonian is finite order in the single-body operators. Thus, only the correlation operator, $S$, needs to be approximated.

The parametrization of the ground bra state ${ }^{11}$ is equally straightforward. The bra-state wave function is parametrized as:

$$
\langle\psi|=\langle\phi| \tilde{S} e^{-S} ; \quad \tilde{S}=1+\sum_{I}^{\prime} \tilde{s}_{I} C_{I},
$$

where $\tilde{S}$ is solely constructed in terms of multiconfigurational destruction operators, defined with respect to the model state $|\phi\rangle$. An arbitrary ground-state observable in the CCM is now completely specified by the set of parameters $\left\{s_{I}, \tilde{s}_{I}\right\}$. An arbitrary expectation value, $\bar{A}$, can thus be expressed as:

$$
\bar{A}=\left\langle\phi\left|\tilde{S} e^{-S} A e^{S}\right| \phi\right\rangle=\bar{A}\left[s_{I}, \tilde{s}_{I}\right] .
$$

In this way all ground-state properties of the system can be obtained.

\section{The CCM Analysis of the Spin-Half $X X Z$ Model Antiferromagnet}

The Hamiltonian for the spin-half $X X Z$ model antiferromagnet, defined with respect to a Néel-ordered ground state, where all the spins have been (mathematically) rotated to the down position, which is the chosen model state, is given by:

$$
H=-\frac{1}{4} \sum_{i, \rho}\left[\frac{\Delta}{2} \sigma_{i}^{z} \sigma_{i+\rho}^{z}+\sigma_{i}^{+} \sigma_{i+\rho}^{+}+\sigma_{i}^{-} \sigma_{i+\rho}^{-}\right],
$$

where $\Delta$ is the anisotropy parameter and the summation is over all the lattice sites, $i$, and nearest neighbors $\rho$. We restrict the values of $\Delta$ to those that produce the AFM ground state, and hence the total $z$-component of spin, $S_{T}^{z}$, must have the value $S_{T}^{z}=0$. To express the $X X Z$ model Hamiltonian in the form shown, a spin rotation has been performed on the up-spin sub-lattice, via the transformation $\sigma^{x} \rightarrow$ $-\sigma^{x}, \sigma^{y} \rightarrow \sigma^{y}, \sigma^{z} \rightarrow-\sigma^{z}$. Equivalently, the single-spin creation and destruction operators are defined as $\sigma^{ \pm} \equiv \frac{1}{2}\left(\sigma^{x} \pm i \sigma^{y}\right)$ on the down-spin sub-lattice, and hence by $\sigma^{ \pm} \equiv \frac{1}{2}\left(-\sigma^{x} \pm i \sigma^{y}\right)$ on the up-spin sub-lattice.

Classically, the spin-half AFM on the honeycomb lattice has perfect Néel ordering for all values $\Delta>1$. Quantum-mechanically, such perfect ordering occurs only in the Ising limit, $\Delta \rightarrow \infty$. The same Hamiltonian of Eq. (8) yields the Heisenberg model at $\Delta=1$ and the $X Y$ model at $\Delta=0$. In order to apply the CCM to a particular case, the correlation operator, $S$, needs to be approximated. Various such truncation schemes are considered below.

\subsection{The full SUB2 scheme}

The particular CCM approximation scheme that will first be implemented here is the so-called full SUB2 scheme, ${ }^{12}$ which includes all two-body correlations built 
on top of the model state $|\phi\rangle$. The full SUB2 correlation operator thus has the following form:

$$
S \rightarrow S_{\mathrm{SUB} 2} \equiv \sum_{\mathbf{p}, \mathbf{q}} S_{\mathbf{p} ; \mathbf{q}} \sigma_{\mathbf{p}}^{+} \sigma_{\mathbf{q}}^{+},
$$

where the vectors $\mathbf{p}$ and $\mathbf{q}$ are vectors from an origin to points on each of the two sub-lattices, respectively. By making use of the lattice symmetries, the correlation operator can thus be alternatively expressed as:

$$
S_{\mathrm{SUB} 2}=\sum_{\mathbf{p}, \mathbf{r}} b_{\mathbf{r}} \sigma_{\mathbf{p}}^{+} \sigma_{\mathbf{p}+\mathbf{r}}^{+}
$$

where the vector $\mathbf{r}$ denotes a vector joining the up-spin sub-lattice to the down-spin sub-lattice.

By applying Eqs. (3) and (4) respectively from the CCM formulation with the defined correlation operator, $S$, the ground-state energy can be shown to be given as follows,

$$
\frac{E_{g}}{N}=-\frac{3}{8}\left(1+2 \Delta b_{1}\right)
$$

and the required nearest-neighbor pair correlation coefficient, $b_{1}$, can be shown to be given by,

$$
b_{1}=\frac{3 \sqrt{3}}{8 \pi^{2}} \int_{C^{\prime}} K\left[1-\sqrt{1-k^{2}|\gamma(\mathbf{q})|^{2}}\right] d \mathbf{q}
$$

where

$$
K \equiv \Delta+2 b_{1}, \quad k^{2} \equiv \frac{1+2 \Delta b_{1}+2 b_{1}^{2}}{\left(\Delta+2 b_{1}\right)^{2}} .
$$

The coefficient $b_{1}$ and the function $\gamma(\mathbf{q})$ are defined as follows,

$$
b_{1}=S_{\mathbf{p} ; \mathbf{p}+\rho} ; \quad \gamma(\mathbf{q}) \equiv \frac{1}{3} \sum_{\rho} e^{i \rho \cdot \mathbf{q}},
$$

where $\rho$ is a nearest neighbor vector joining any site on the honeycomb lattice to any one of its three nearest neighbors. The integral in Eq. (12) is performed over the reciprocal lattice primitive cell $C^{\prime}$. Details of the derivation are given in Appendix A. The full SUB2 ground-state energy per spin for the Heisenberg model is given in Table 1.

Table 1. The ground-state energy per spin at the Heisenberg point, $\Delta=1$, under various CCM schemes, compared with the results of a Monte Carlo simulation, ${ }^{6}$ and of a series expansion. ${ }^{8}$

\begin{tabular}{ccccc}
\hline full SUB2 & LSUB4 & full SUB2+LSUB4 & Series Expansion & Monte Carlo \\
\hline-0.528 & -0.532 & -0.537 & -0.547 & $-0.545 \pm 0.001$ \\
\hline
\end{tabular}


Equation (12) is a self-consistent relation from which a terminating point can be obtained, as the factor inside the square root can be shown to be negative for values of the anisotropy, $\Delta$, less than some terminating value, $\Delta_{\mathrm{c}}$. The computed SUB2 terminating value is at $\Delta_{\mathrm{c}} \simeq 0.70983$. There are indications that at this point, within the SUB2 approximation, there may be a phase transition. Primarily, evidence for this comes from the asymptotic behavior of the ket state correlation coefficient, as $|\mathbf{r}| \rightarrow \infty$ :

$$
b_{\mathrm{r}} \rightarrow\left\{\begin{array}{lc}
O\left[\frac{1}{l|\mathbf{r}|}\right], & \Delta>\Delta_{\mathrm{c}}, \\
\frac{0.0015}{|\mathbf{r}|^{2}}, & \Delta=\Delta_{c},
\end{array}\right.
$$

where $b_{\mathbf{r}} \equiv S_{\mathbf{p} ; \mathbf{p}+\mathbf{r}}$, and where the vector $\mathbf{r}$ joins a site from one of the two equivalent sub-lattices to the other. The spin-spin correlation function can be expressed in terms of the full SUB2 correlation coefficient. ${ }^{10}$ The changeover from exponential to algebraic decay of the ket-state correlation coefficient, $b_{\mathbf{r}}$, at the terminating point $\Delta_{\mathrm{c}} \simeq 0.70983$ is thus seen to be characteristic of the expected behavior at a phase transition.

The anisotropy susceptibility defined as:

$$
A \equiv \frac{d^{2} E}{d \Delta^{2}}
$$

where $E \equiv E_{\mathrm{g}} / N$ is the ground-state energy per spin, provides further evidence of a phase change at the full SUB2 terminating point. A phase transition is characterized by singular behavior of the thermodynamic quantities at the critical point. As $\Delta \rightarrow$ $\Delta_{c}$, the anisotropy susceptibility, $A$, tends to infinity, therefore exhibiting singular behavior, as shown in Fig. 1. Thus, there is strong evidence that the terminating point may correspond, within the limitations of the SUB2 approximation, to the physical transition point of the Hamiltonian between Ising-like to $X Y$-like phases.

As stated earlier, the sub-lattice magnetization can also be calculated. Details of the technique have been published elsewhere. ${ }^{11} \mathrm{~A}$ comparison of results appears in Table 2, and Fig. 2 exhibits the full SUB2 terminating point for the sub-lattice magnetization.

Table 2. The staggered magnetization as a fraction of the saturation value, from a Monte Carlo simulation, ${ }^{6}$ a finite lattice diagonalization, ${ }^{7}$ a series expansion calculation, ${ }^{8}$ and a spin-wave theory (SWT) calculation, ${ }^{9}$ at the Heisenberg point. The CCM full SUB2 result is given at $\Delta_{c}$.

\begin{tabular}{ccccc}
\hline CCM full SUB2 & Series Expansion & SWT & Finite Lattice Diag. & Monte Carlo \\
\hline 0.52 & 0.52 & 0.5 & 0.46 & 0.44 \\
\hline
\end{tabular}




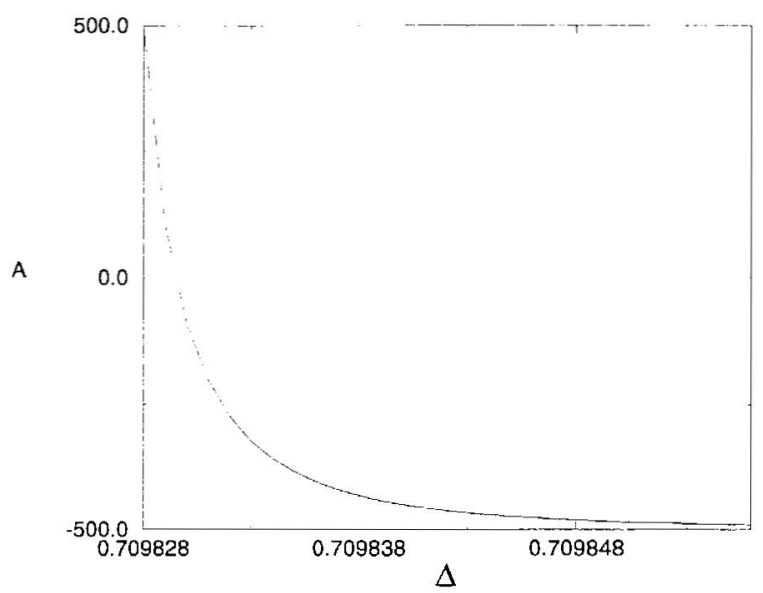

Fig. 1. The anisotropy susceptibility, $A$, of the spin-half $X X Z$ model on the honeycomb lattice as a function of the anisotropy, $\Delta$, near the terminating point, from the results of the full SUB2 approximation scheme.

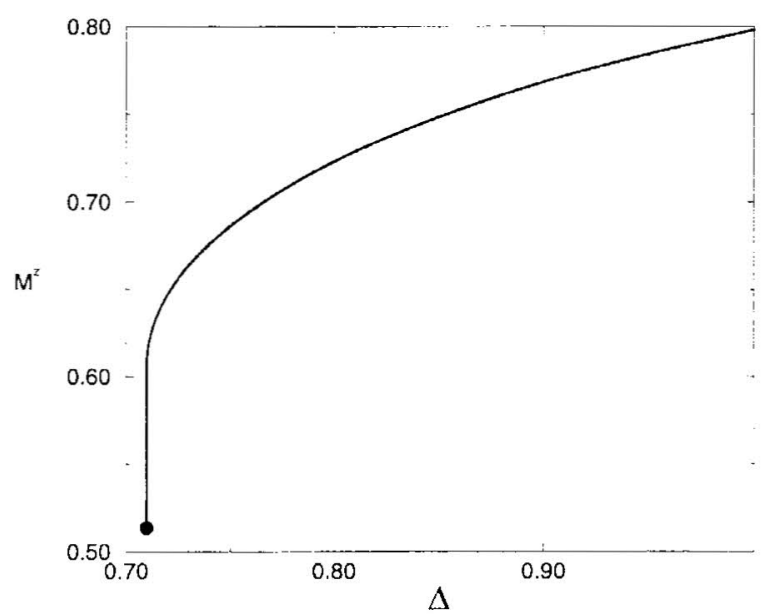

Fig. 2. The sub-lattice magnetization, $M^{z}$, for the spin-half $X X Z$ model on the honeycomb lattice, as a function of the anisotropy, $\Delta$, from the results of the full SUB2 scheme. The termination point is shown by the solid circle.

\subsection{The LSUB4 scheme}

The localized LSUB4 scheme retains up to and including four-body correlations, but restricted only to those which occur over all configurations of four adjacent lattice sites. The full SUB4 correlation operator, $S$, is expressed as:

$$
S_{\mathrm{SUB} 4} \equiv \sum_{\mathbf{p}, \mathbf{q}} S_{\mathbf{p} ; \mathbf{q}} \sigma_{\mathbf{p}}^{+} \sigma_{\mathbf{q}}^{+}+\frac{1}{4} \sum_{\mathbf{p}, \mathbf{p}^{\prime}} \sum_{\mathbf{q}, \mathbf{q}^{\prime}} S_{\mathbf{p}, \mathbf{p}^{\prime} ; \mathbf{q}, \mathbf{q}^{\prime}} \sigma_{\mathbf{p}}^{+} \sigma_{\mathbf{q}}^{+} \sigma_{\mathbf{p}^{\prime}}^{+} \sigma_{\mathbf{q}^{\prime}}^{-} .
$$



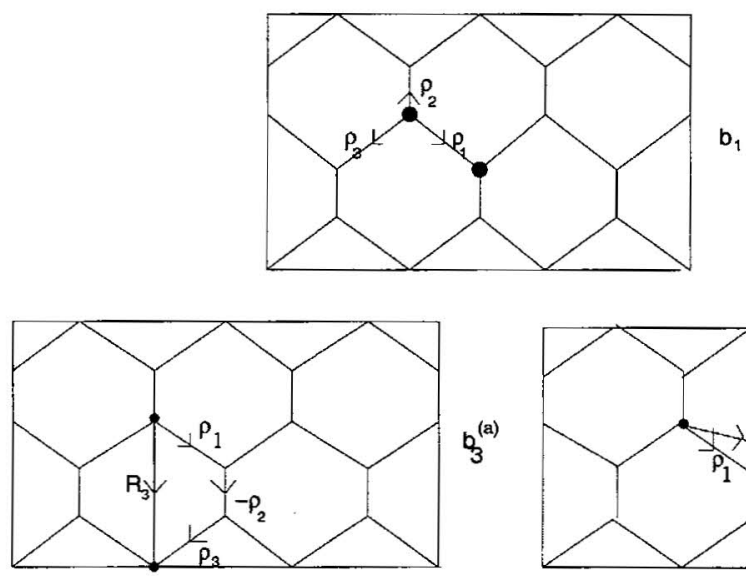

$\xi^{(a)}$
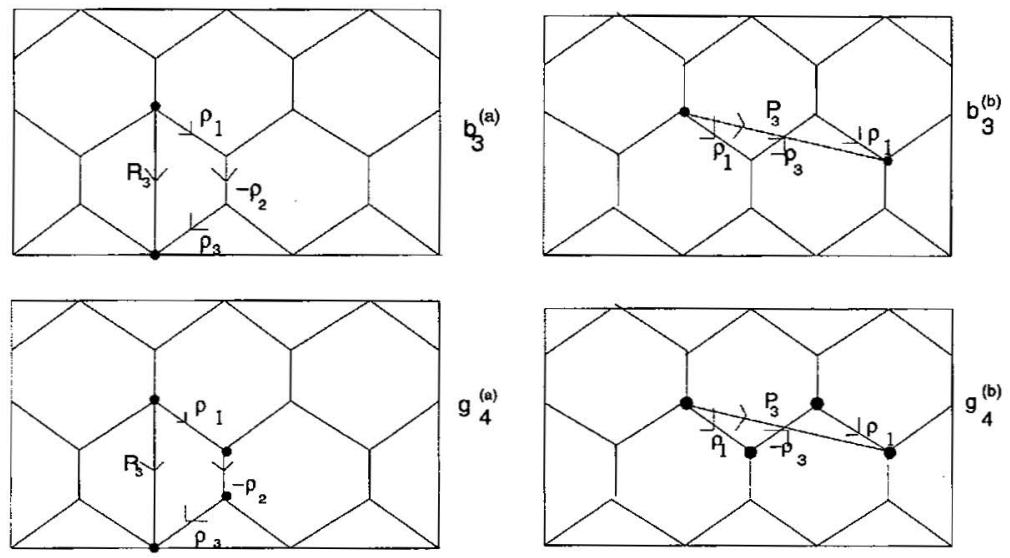

Fig. 3. An illustration of the five fundamental configurations present in the 2D LSUB4 scheme on the honeycomb lattice. The dots indicate the flipped spins with respect to the model Néel state.

If the symmetries in the honeycomb lattice, as well as the restriction $S_{T}^{z}=0$, are taken into account, there are only five independent LSUB4 configurations, as shown in Fig. 3. Therefore, the approximated correlation operator is defined schematically as follows:

$$
\begin{aligned}
S \rightarrow S_{\mathrm{LSUB} 4}= & b_{1} \sum_{\mathbf{p}, \rho} \sigma_{\mathbf{p}}^{+} \sigma_{\mathbf{p}+\rho}^{+}+b_{3}^{(a)} \sum_{\mathbf{p}, \mathbf{R}} \sigma_{\mathbf{p}}^{+} \sigma_{\mathbf{p}+\mathbf{R}}^{+}+b_{3}^{(b)} \sum_{\mathbf{p}, \mathbf{P}} \sigma_{\mathbf{p}}^{+} \sigma_{\mathbf{p}+\mathbf{P}}^{+} \\
& +g_{4}^{(a)} \sum_{\mathbf{p}, \rho, v, \mathbf{R}} \sigma_{\mathbf{p}}^{+} \sigma_{\mathbf{p}+\rho}^{+} \sigma_{\mathbf{p}+v}^{+} \sigma_{\mathbf{p}+\mathbf{R}}^{+}+g_{4}^{(b)} \sum_{\mathbf{p}, \rho, v, \mathbf{P}} \sigma_{\mathbf{p}}^{+} \sigma_{\mathbf{p}+\rho}^{+} \sigma_{\mathbf{p}+v}^{+} \sigma_{\mathbf{p}+\mathbf{P}}^{+},
\end{aligned}
$$

where the vector $\rho$ denotes the set of three nearest-neighbor vectors $\rho=\left\{\rho_{1}, \rho_{2}, \rho_{3}\right\}$. The sets $\mathbf{R}, \mathbf{P}$ and $v$, denote respectively the vectors $\mathbf{R}=\left\{\mathbf{R}_{1}, \mathbf{R}_{2}, \mathbf{R}_{3}\right\}$, with three symmetries, $\mathbf{P}=\left\{\mathbf{P}_{1}, \mathbf{P}_{2}, \mathbf{P}_{3}, \mathbf{P}_{4}, \mathbf{P}_{4}, \mathbf{P}_{5}, \mathbf{P}_{6}\right\}$, with six symmetries, and $v=$ $\left\{v_{1}, v_{2}, v_{3}, v_{4}, v_{5}, v_{6}\right\}$, with six symmetries in the lattice, as shown in Fig. 4.

From Eq. (4) the correlation operator produces five independent coupled equations, which need to be solved numerically in order to obtain the ground-state energy,

$$
\begin{aligned}
0= & 1-4 \Delta b_{1}-5 b_{1}^{2}+4 b_{1} b_{3}^{(a)}+4 b_{1} b_{3}^{(b)}+2 b_{3}^{2(a)}+4 b_{3}^{2(b)} \\
& +8 b_{3}^{(a)} b_{3}^{(b)}+4 g_{4}^{(a)}+4 g_{4}^{(b)}
\end{aligned}
$$



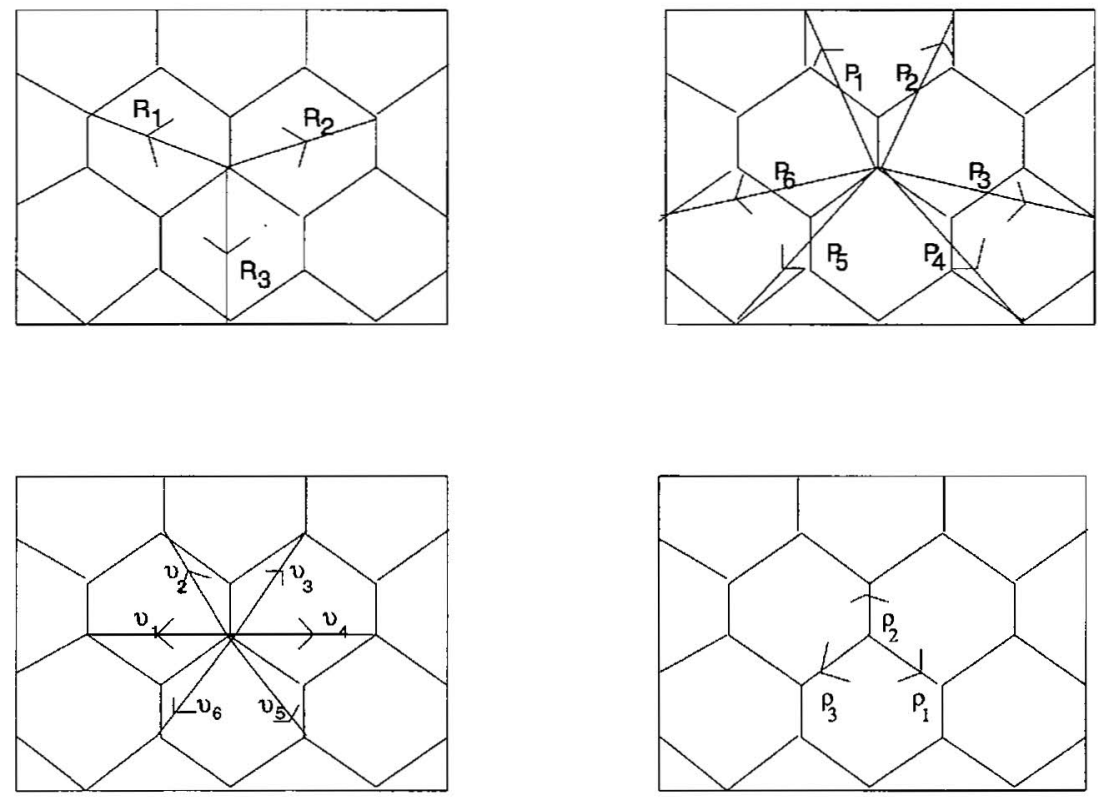

Fig. 4. An illustration of the vectors belonging to the sets, $\mathbf{R}, \mathbf{P}, v$, and $\rho$ respectively.

$$
\begin{aligned}
0= & -6 \Delta b_{3}^{(a)}-6 b_{1} b_{3}^{(a)}+4 b_{1} b_{3}^{(b)}+6 b_{3}^{(b) 2}+b_{1}^{2}+4 b_{3}^{(a)} b_{3}^{(b)}+2 g_{4}^{(a)} \\
0= & -6 \Delta b_{3}^{(b)}-4 b_{1} b_{3}^{(b)}+2 b_{1} b_{3}^{(a)}+2 b_{3}^{(a) 2}+2 b_{3}^{(b) 2}+b_{1}^{2} \\
& +2 b_{3}^{(a)} b_{3}^{(b)}+g_{4}^{(b)} \\
0= & \Delta\left(3 g_{4}^{(a)}-2 b_{1} b_{3}^{(a)}-b_{1}^{2}\right)+5 b_{1} g_{4}^{(a)}+b_{1} g_{4}^{(b)}+b_{3}^{(a)} g_{4}^{(a)} \\
& -b_{3}^{(b)} g_{4}^{(b)}-b_{3}^{(b)} g_{4}^{(a)}+2 b_{1} b_{3}^{(a)} b_{3}^{(b)}+2 b_{1} b_{3}^{(a) 2}+2 b_{1}^{2} b_{3}^{(b)} \\
0= & \Delta\left(3 g_{4}^{(b)}-2 b_{1} b_{3}^{(b)}-b_{1}^{2}\right)+6 b_{1} g_{4}^{(b)}+b_{1} g_{4}^{(a)}+2 b_{1} b_{3}^{(b) 2} \\
& -2 b_{3}^{(a)} g_{4}^{(a)}-b_{3}^{(b)} g_{4}^{(a)}+2 b_{1} b_{3}^{(a)} b_{3}^{(b)}+2 b_{1} b_{3}^{2(a)}+2 b_{1}^{2} b_{3}^{(a)}
\end{aligned}
$$

Numerically, we find that the configuration responsible for most of the quantum fluctuations present in this case is the $b_{1}$ configuration in Fig. 3 . The other four configurations are of roughly equal importance. Results for the LSUB4 approximation are shown in Table 1.

\subsection{The full SUB2+LSUB4 scheme}

The results for the ground-state energy from the full SUB2 scheme are comparable with those from the LSUB4 scheme. Thus, we are led to suspect that the full SUB2 scheme can be improved by additionally taking into account the localized 
four-body corrections from the LSUB4 scheme. The resultant full SUB2+LSUB4 approximation scheme might also be expected to produce a more accurate value for the terminating point than that from the full SUB2 scheme. From the CCM formulation in Eq. (4) a set of six equations is obtained. Those equations are again solved numerically to find the ground-state energy:

$$
\begin{aligned}
& 0=1-4 \Delta b_{1}-10 b_{1}^{2}+\sum_{\mathbf{r}^{\prime}, \rho} b_{\mathbf{r}^{\prime}} b_{-\mathbf{r}^{\prime}+\rho_{1}+\rho}+4 g_{4}^{(a)}+4 g_{4}^{(b)}, \quad \mathbf{r}=\rho_{1}, \\
& 0=-6 \Delta b_{3}^{(a)}-12 b_{1} b_{3}^{(\alpha)}+\sum_{\mathbf{r}^{\prime}, \rho} b_{\mathbf{R}_{1}+\mathbf{r}^{\prime}-\rho_{1}} b_{-\mathbf{r}^{\prime}+\rho_{1}+\rho}+2 g_{4}^{(a)}, \quad \mathbf{r}=\mathbf{R}_{1} \\
& 0=-6 \Delta b_{3}^{(b)}-12 b_{1} b_{3}^{(b)}+\sum_{\mathbf{r}^{\prime}, \rho} b_{\mathbf{P}_{1}+\mathbf{r}^{\prime}-\rho_{1}} b_{-\mathbf{r}^{\prime}+\rho_{1}+\rho}+g_{4}^{(b)}, \quad \mathbf{r}=\mathbf{P}_{1} \\
& 0=-6\left(\Delta+2 b_{1}\right) b_{\mathbf{r}}+\sum_{\mathbf{r}^{\prime}, \rho} b_{\mathbf{r} \div \mathbf{r}^{\prime}-\rho_{1}} b_{-\mathbf{r}^{\prime}+\rho_{1}+\rho}, \quad|\mathbf{r}|>\left|\mathbf{R}_{1}\right|,\left|\mathbf{P}_{1}\right| \\
& 0=\Delta\left(3 g_{4}^{(a)}-2 b_{1} b_{3}^{(a)}-b_{1}^{2}\right)+5 b_{1} g_{4}^{(a)}+b_{1} g_{4}^{(b)}+b_{3}^{(a)} g_{4}^{(a)} \\
& -b_{3}^{(b)} g_{4}^{(b)}-b_{3}^{(b)} g_{4}^{(a)}+2 b_{1} b_{3}^{(a)} b_{3}^{(b)}+2 b_{1} b_{3}^{(a) 2}+2 b_{1}^{2} b_{3}^{(b)} \\
& 0=\Delta\left(3 g_{4}^{(b)}-2 b_{1} b_{3}^{(b)}-b_{1}^{2}\right)+6 b_{1} g_{4}^{(b)}+b_{1} g_{4}^{(a)}+2 b_{1} b_{3}^{(b) 2} \\
& -2 b_{3}^{(a)} g_{4}^{(a)}-b_{3}^{(b)} g_{4}^{(a)}+2 b_{1} b_{3}^{(a)} b_{3}^{(b)}+2 b_{1} b_{3}^{2(a)}+2 b_{1}^{2} b_{3}^{(a)},
\end{aligned}
$$

where $b_{\mathbf{R}} \equiv b_{3}^{(a)}$ and $b_{\mathbf{P}} \equiv b_{3}^{(b)}$, as shown in Fig. 5

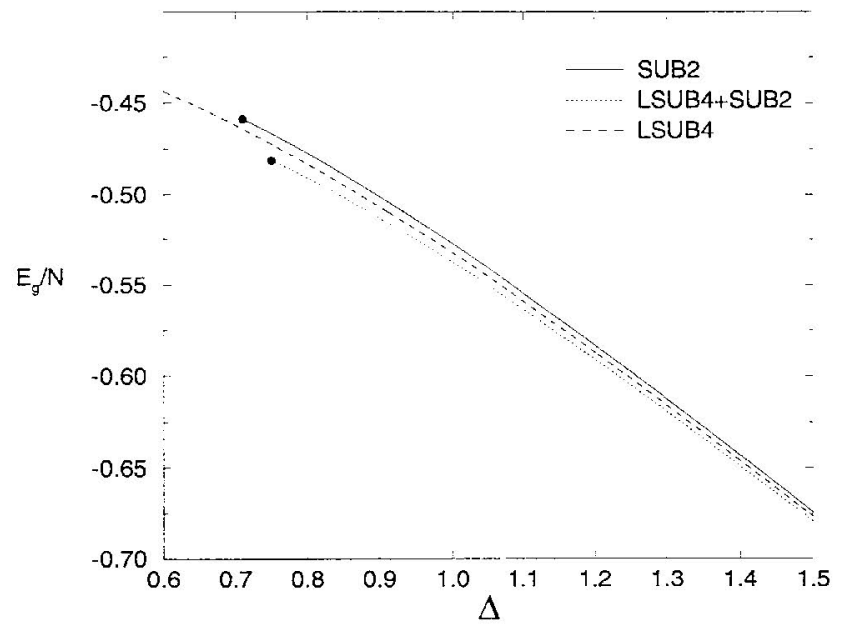

Fig. 5. The ground-state energy per spin for the spin-half $X X Z$ model on the honeycomb lattice, as a function of the anisotropy, $\Delta$, from the results of the LSUB4, full SUB2, and full SUB2+LSUB4 approximation schemes. The solid circles indicate the respective termination points, $\Delta_{c}$. 
As expected, this approximation scheme produces the most accurate value for the ground-state energy, of the three CCM schemes implemented here, as shown in Table 1. The full SUB2+LSUB4 value for the terminating point, at $\Delta_{\mathrm{c}} \simeq 0.746$, is also closer to the expected value of unity than the corresponding full SUB2 result at $\Delta_{\mathrm{c}} \simeq 0.710$.

\section{Discussion and Conclusion}

In this paper, various CCM approximation schemes have been implemented successfully for the particular case of the spin-half $X X Z$ model on the honeycomb lattice. From the full SUB2 scheme, there is evidence of a phase transition in this model, because this approximation scheme includes arbitrarily long-range twobody correlations. The LSUB4 scheme also produces reasonably good values for the ground-state energy. Thus, the full SUB2+LSUB4 scheme combines the best features of both these schemes. It obtains improved estimates for both the groundstate energy and the terminating point. This demonstrates the capacity of the CCM for systematic improvement.

Application of the CCM to the honeycomb lattice case produces results which are in accordance with the expected behavior of this model. The honeycomb lattice has the smallest co-ordination number of any $2 \mathrm{D}$ lattice. Thus, quantum fluctuations in the honeycomb lattice should be greater than in the square lattice case but less than in the $1 \mathrm{D}$ chain. However, unlike the $1 \mathrm{D}$ chain case, LRO survives. Therefore, the low-order approximation schemes, implemented here, obtain the qualitative behavior of the spin-half honeycomb case.

The CCM has again been seen to be capable of obtaining some critical properties of this model. The algebraic decay of the correlation coefficients and the singular behavior of the anisotropy susceptibility at the full SUB2 terminating point are characteristics of a phase transition. The ability of such an $a b$ initio method to yield evidence, even if not completely conclusive, of phase transitions, and to give accurate values of the energy and staggered magnetization, for values of the anisotropy parameter over a wide range, including the phase transition point, is certainly encouraging.

The CCM ground-state energies and sub-lattice magnetization, given in Tables 1 and 2 respectively, are not yet at the same level of accuracy as the Monte Carlo simulations. However, for such low-order approximations, reasonable results are obtained for very little computing time. However, we also note that results of the same order of accuracy as Monte Carlo simulations have been obtained for the $X X Z$ model on other lattices from high-order CCM schemes. ${ }^{12}$ Therefore, the future for the CCM must lie in high-order approximation schemes, in order to produce results at least as good as Monte Carlo simulations ${ }^{12}$ and to investigate critical behavior in more depth. Such high-order schemes can be implemented through the use of computer algebra. Work along these lines is currently in progress. So far, highorder results for anisotropic spin-half antiferromagnets have only been obtained 
for the one-dimensional chain ${ }^{13}$ and for the two-dimensional square and triangular lattices. ${ }^{14}$ Reference 14 describes a rather general procedure for implementing the CCM to high orders for a broad class of spin lattice models.

\section{Acknowledgments}

One of us (RFB) gratefully acknowledges the support for this work in the form of a grant from the Engineering and Physical Sciences Research Council (EPSRC) of Great Britain.

\section{Appendix A}

The ground ket-state equation, in the CCM formulation, is obtained from Eq. (4) by using the full SUB2 correlation operator of $\mathrm{Eq}$. (10) to give:

$$
0=\sum_{\rho}\left[\left(1+2 \Delta b_{1}+2 b_{1}^{2}\right) \delta_{\rho, \mathbf{r}}-2\left(\Delta+2 b_{1}\right) b_{\mathbf{r}}+\sum_{\mathbf{r}^{\prime}} b_{\mathbf{r}^{\prime}} b_{\mathbf{r}-\mathbf{r}^{\prime}+\rho}\right]
$$

where the sum over $\rho$ runs over the three nearest neighbor lattice vectors. The indices $\mathbf{r}$ and $\mathbf{r}^{\prime}$ belong to the set $\left\{\mathbf{r}_{i, j}\right\}$, where $\mathbf{r}_{i, j}=\mathbf{x}_{i}-\mathbf{x}_{j}$ for $\mathbf{x}_{i}$ and $\mathbf{x}_{j}$ the co-ordinates of a site on the $i$-sub-lattice and the $j$-sub-lattice, respectively. From the above definition of the vectors, $\mathbf{r}$, the appropriate transform is a sub-lattice Fourier-transform, which is defined for the coefficients, $b_{\mathbf{r}}$, by the relation:

$$
B(\mathbf{q}) \equiv \sum_{\mathbf{r}} e^{i \mathbf{r} \cdot \mathbf{q}} b_{\mathbf{r}}
$$

where $\mathbf{q}=\left(q_{x}, q_{y}\right)$ are the points in the Brillouin zone on the sub-lattice, and the sum on $r$ runs over all distinct sub-lattice vectors which join points on opposite spin sub-lattices. Thus, the Fourier-transformed ket-state equation is given by:

$$
0=\left(1+2 \Delta b_{1}+2 b_{1}^{2}\right) \gamma(\mathbf{q})-2\left(\Delta+2 b_{1}\right) B(\mathbf{q})+\gamma(-\mathbf{q}) B^{2}(\mathbf{q})
$$

where

$$
\gamma(\mathbf{q}) \equiv \frac{1}{3} \sum_{\rho} e^{i \rho \cdot \mathbf{q}}=\frac{1}{3}\left(e^{i q_{y}}+2 e^{-\frac{i}{2} q_{y}} \cos \frac{\sqrt{3}}{2} q_{x}\right) .
$$

Equation (A.3) is of the form of a quadratic equation. It can be solved simply as follows:

$$
B(\mathbf{q})=\frac{K \pm K \sqrt{1-k^{2} \gamma(-\mathbf{q}) \gamma(\mathbf{q})}}{\gamma(-\mathbf{q})}
$$

where

$$
K \equiv \Delta+2 b_{1} ; \quad k^{2} \equiv \frac{1+2 \Delta b_{1}+2 b_{1}^{2}}{\left(\Delta+2 b_{1}\right)^{2}} .
$$

As $\Delta \rightarrow \infty$ in the Ising limit, the quantum fluctuations are required to disappear. Thus, each of the sub-lattice coefficients, $b_{\mathbf{r}}$, vanishes as $\Delta \rightarrow \infty$. In particular, $b_{1} \rightarrow 0$. Thus, $k^{2} \rightarrow 0$ in this limit, and hence the positive sign of the square 
root must be discarded. Now, in order to calculate the ground-state energy from Eq. (11), it is necessary to invert Eq. (A.2). This is possible, since only the sublattice is important in the calculation and even though the honeycomb lattice itself is non-Bravais, its sub-lattices in both the direct and reciprocal space are Bravais. Hence,

$$
b_{\mathbf{r}}=\frac{1}{V} \int_{C^{\prime}} e^{-i \mathbf{r} \cdot \mathbf{q}} B(\mathbf{q}) d \mathbf{q},
$$

where $V$ is the volume of the reciprocal primitive lattice cell and the integration is performed over the reciprocal lattice primitive cell $C^{\prime}$. So, the above integral can be written explicitly as:

$$
b_{\mathbf{r}}=\frac{3 \sqrt{3}}{8 \pi^{2}} \int_{C^{\prime}} e^{-i \mathbf{r} \cdot \mathbf{q}} B(\mathbf{q}) d \mathbf{q} .
$$

In order to obtain the coefficient $b_{1}$, we let $\mathbf{r} \rightarrow \rho$ in the above equation. Thus, $b_{1}$ is given by:

$$
\begin{aligned}
b_{1} & =\frac{3 \sqrt{3}}{8 \pi^{2}} \int_{C^{\prime}} e^{-i \rho \cdot \mathbf{q}} B(\mathbf{q}) d \mathbf{q} \\
& \equiv \frac{3 \sqrt{3}}{8 \pi^{2}} \int_{C^{\prime}} \gamma(-\mathbf{q}) B(\mathbf{q}) d \mathbf{q}
\end{aligned}
$$

Equation (A.4) can now be substituted into Eq. (A.8) to give:

$$
b_{1}=\frac{3 \sqrt{3}}{8 \pi^{2}} \int_{C^{\prime}} K\left[1-\sqrt{1-k^{2}|\gamma(\mathbf{q})|^{2}}\right] d \mathbf{q},
$$

where each vector $\mathbf{q}$ is in the magnetic zone of the honeycomb lattice, which is hexagonal. However, the sub-lattice reciprocal vectors form a rhombus, which is a primitive cell with the full symmetry of the Bravais hexagonal sub-lattice. Therefore, the vectors $q$ can be easily defined within the rhombus. The reciprocal lattice vectors are given by:

$$
\mathbf{a}=2 \pi\left(\frac{1}{3}, \frac{1}{\sqrt{3}}\right) ; \quad \mathbf{b}=2 \pi\left(\frac{2}{3}, 0\right) .
$$

The vector $\mathbf{q}$ is defined by:

$$
\mathbf{q}=\frac{m_{1}}{N_{1}} \mathbf{a}+\frac{m_{2}}{N_{2}} \mathbf{b} ; \quad 0 \leq \frac{m_{1}}{N_{1}}, \frac{m_{2}}{N_{2}} \leq 1
$$

where $m_{1}$ and $m_{2}$ are integers. Hence, $0 \leq m_{1}, m_{2} \leq \sqrt{N / 2}-1$, as there are $N / 2$ lattice sites in the sub-lattice. Thus, the Cartesian $x$ and $y$ components of the vector $\mathbf{q}$ are given by:

$$
q_{x}=\frac{2 \sqrt{2} \pi}{3 \sqrt{N}}\left(m_{1}+2 m_{2}\right) ; \quad q_{y}=\frac{2 \sqrt{2} \pi}{\sqrt{3 N}} m_{1} .
$$


In order to calculate the integral (A.9), it must be discretized. Firstly, the integral is transformed in terms of the variables $m_{1}$ and $m_{2}$ in the following way:

$$
\int d q_{x} d q_{y}=\int d m_{1} d m_{2}|J|
$$

where $|J|=16 \pi^{2} / 3 \sqrt{3} N$ is the determinant of the Jacobian matrix. Thus, the integral (A.9) can be expressed as:

$$
b_{1}=\frac{2}{N} \int_{C^{\prime}} K\left[1-\sqrt{1-k^{2} \mid \gamma\left(\mathbf{q}\left(m_{1}, m_{2}\right)\right)^{2}}\right] d m_{1} d m_{2}
$$

This integral can now be easily discretized as:

$$
b_{1} \simeq \frac{2}{N} \sum_{m_{1}, m_{2}} K\left[1-\sqrt{1-k^{2}\left|\gamma\left(\mathbf{q}\left(m_{1}, m_{2}\right)\right)\right|^{2}}\right] .
$$

Finally, the ground-state energy can be obtained from Eq. (11).

\section{References}

1. H. A. Bethe, Z. Phys. 71, 205 (1931).

2. E. Manousakis, Rev. Mod. Phys. 63, 1 (1991).

3. C. Zeng, I. Staples and R. F. Bishop, J. Phys.: Condens. Matter 7, 9021 (1995).

4. P. W. Anderson Phys. Rev. 86, 694 (1952); R. Kubo, ibid. 87, 568 (1952).

5. T. Oguchi, Phys. Rev. 117, 117 (1960).

6. J. D. Reger, J. A. Riera and A. P. Young, J. Phys.: Condens. Matter 1, 1855 (1989).

7. J. Oitmaa and D. D. Betts, Can. J. Phys. 56, 897 (1978); M. Suzuki and S. Miyashita, ibid. 56, 902 (1978).

8. J. Oitmaa, C. J. Hamer and W. H. Zheng, Phys. Rev. B45, 9834 (1992).

9. W. H. Zheng, J. Oitmaa and C. J. Hamer, Phys. Rev. B44, 11869 (1991).

10. R. F. Bishop, J. B. Parkinson and Y. Xian, Phys. Rev. B43, 13782 (1991).

11. R. F. Bishop and H. Kümmel, Phys. Today 40(3), 52 (1987); R. F. Bishop, Theor. Chim. Acta. 80, 95 (1991).

12. R. F. Bishop, R. G. Hale and Y. Xian, Phys. Rev. Lett. 73, 3157 (1994).

13. R. F. Bishop, R. G. Hale and Y. Xian, Int. J. Quantum Chem. 57, 919 (1996).

14. C. Zeng, D. J. J. Farnell and R. F. Bishop, J. Stat. Phys. 90, 327 (1998). 